\title{
洲 Inserm Immunosenescence of Skin Resident T-Cells
}

Institut national

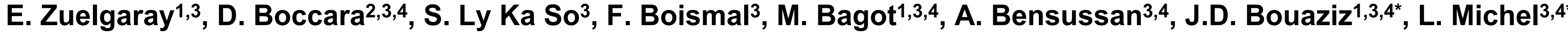 \\ ${ }^{1}$ Dermatology Department, ${ }^{2}$ Plastic and Reconstructive Surgery Department, ${ }^{3}$ INSERM UMRS-976, Saint-Louis Hospital, ${ }^{4}$ Paris-Diderot University, Paris, France \\ * Shared senior authorship}

\section{Background/Objective}

"Immunosenescence" is the impaired immune response associated with normal aging, contributing to an increased susceptibility to infectious diseases and cancer.

Skin immunosenescence is characterized by a decreased number of Langerhans cells, an increased number of regulatory CD4 ${ }^{+} \mathrm{FOXP} 3^{+} \mathrm{T}-$ cells and an overexpression of the inhibitory marker PD1 on CD4 ${ }^{+}$lymphocytes surface with age.

The aim of this work was to characterize the immunosenescence profile of human skin resident conventional T lymphocytes and the innate lymphoid cell population.

\section{Study population}

\section{Material and Methods}

Skin samples from healthy young $(<30$ years; $n=6)$ and old subjects ( $>60$ years; $n=6)$ were obtained from the plastic and reconstructive department of Saint-Louis hospital (mammary reduction surgery or face lift surgery).

\section{Skin samples}

Samples were cultured at $37^{\circ} \mathrm{C}$ in a complete RPMI medium (Roswell Park Memorial Institute) prepared from RPMI 1640 (Sigma-Aldrich) supplemented with 10\% Fetal Bovine Serum (FBS), 1\% glutamine and 1\% penicillin/streptomycin. The lymphocytes present in the supernatant were recovered and filtered after 21 days of culture.

\section{Flow cytometric analysis}

Flow cytometric analysis was performed using BD LSRFortessa ${ }^{\mathrm{TM}}$ (sixteen colors, BD Bioscience).

Data were analyzed with BD FACSDIVA ${ }^{T M}$ software (BD Biosciences).

\section{Results}

No alteration of the percentages of skin $\mathrm{CD}^{+}, \mathrm{CD} 4^{+}$or $\mathrm{CD} 8^{+} \mathrm{T}$-cells with age

Decreased frequency of skin CD45RA ${ }^{+}$naive T-cells with age $(p<0.05)$

Upregulation of CD39 expression on $\mathrm{CD}^{+}{ }^{+} \mathrm{CD} 4^{+} \mathrm{T}$-cells surface in old skin $(p<0.05)$

Upregulation of PD1 expression on skin $C D 3^{+} C D 4^{+}$T-cells surface with age $(p<0.05)$

No alteration of the percentages of skin $\gamma / \delta$ T lymphocytes with age

Decreased number of skin ILC2 with age $(p<0.05)$

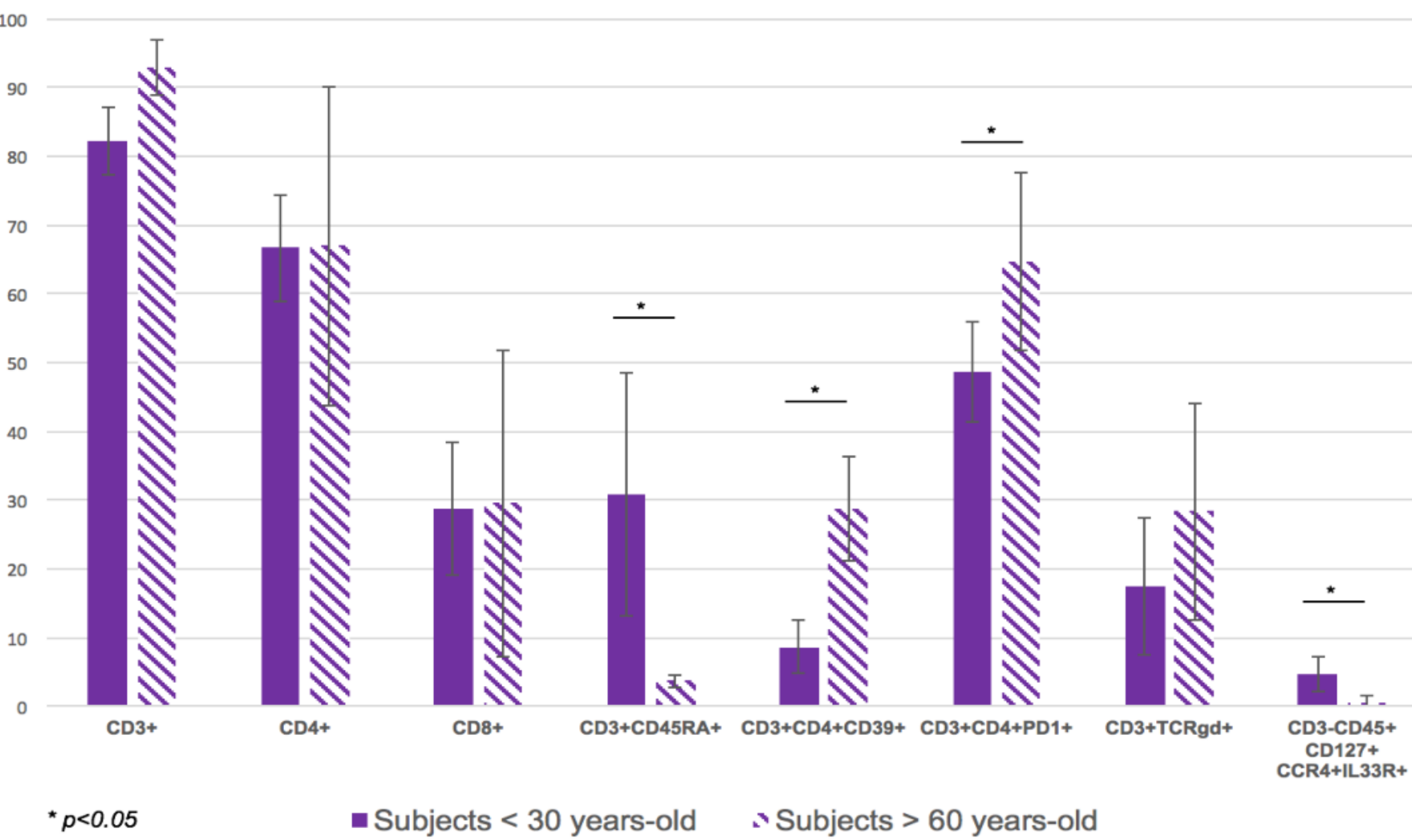

Figure 1: mean expression $( \pm S D, n=6$ ) of surface markers by skin resident $T$ lymphocytes in young and old subjects
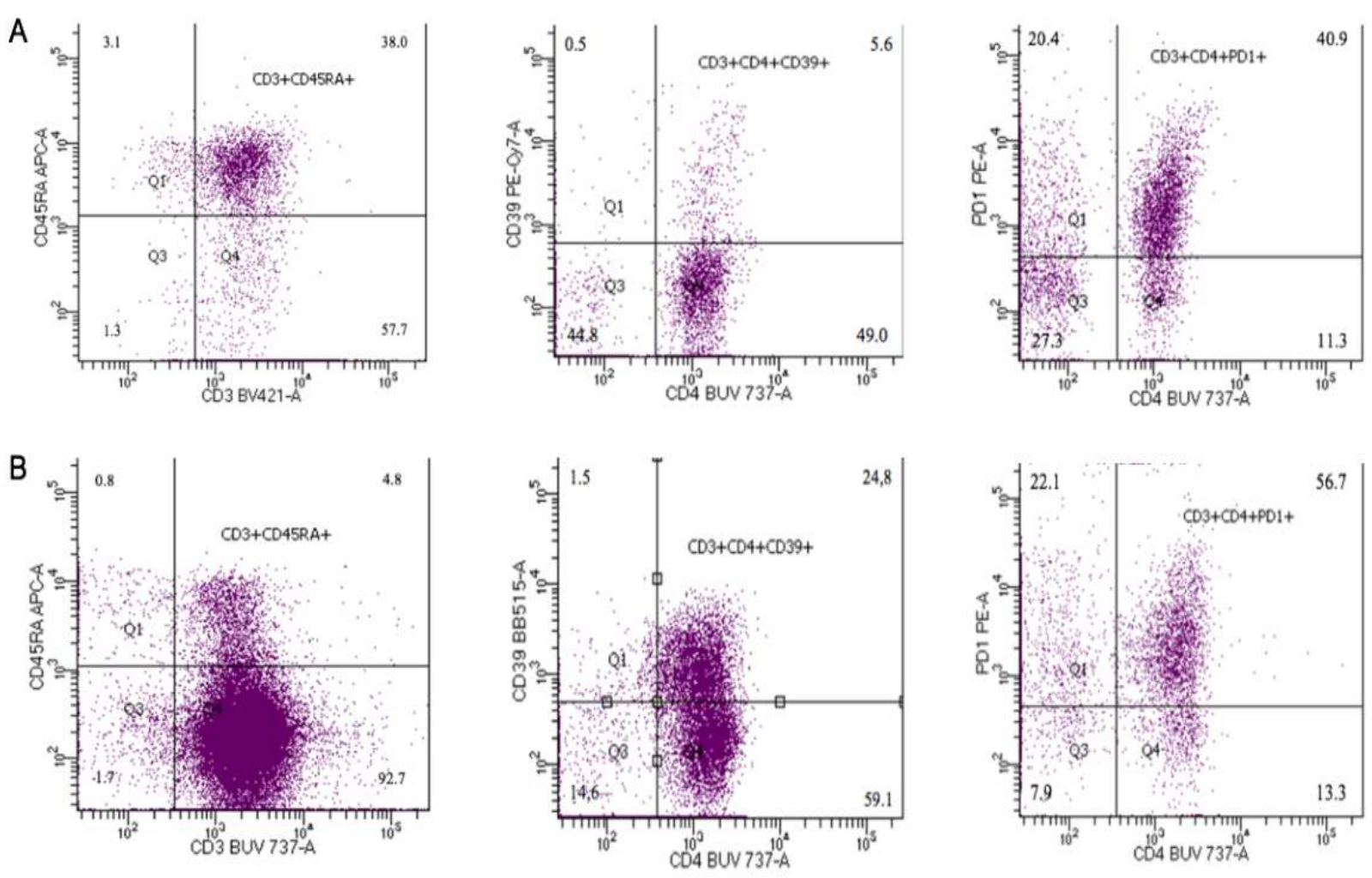

Figure 2: modified phenotype of skin resident T-cells with age in a representative young (A) and elderly subject (B) after flow cytometric analysis

\section{Conclusion}

Upregulation of CD39 and PD1 expression on skin T-cells surface and decreased proportion of skin naïve T-cells with age could contribute to reduced generation of skin long-lived memory T-cells resulting in a lower response against infectious disease, an impaired vaccine response, and a decreased anti-tumoral response.

Decreased number of skin resident ILC2 could explain the decreased incidence of atopic dermatitis in elderly patients. 